\title{
A Preliminary Study on the Humanized Design of Rest Facilities in Open-air Stadium
}

\author{
Wei-hui HUANG ${ }^{1,2}$, Ming CHEN ${ }^{1,2, a}$, Jian-hua LYU ${ }^{1,2, b, *}$ \\ ${ }^{1}$ Forestry College, Sichuan Agricultural University, Chengdu 611130, China \\ ${ }^{2}$ Key Laboratory of Wood Industry and Furniture Engineering of Sichuan Provincial Colleges and \\ Universities, Sichuan Agricultural University, Chengdu 611130, China \\ achenming@sicau.edu.cn, bljh@sicau.edu.cn \\ *Corresponding author
}

Keywords: stadium, rest, facilities, humanization.

\begin{abstract}
Based on the background of the national fitness campaign in recent years, this paper analyzes the research status of the rest facilities in the stadium and facilities' impact on the users, therefore a further thought are to be given to the design of the rest facilities. According to the principle of humanization and with the design evidence, seats for different users have designed and new ideas for the rest facilities design have been provided.
\end{abstract}

\section{Introduction}

With the increasing development of society, people's psychological pressure continues to increase with a wide-ranged population flow, which accelerates pace of life and results in other phenomena ${ }^{[1]}$. People are constantly concerned about health, thus their sports awareness gradually increased. With the evolution of social sports and the popular fitness craze, people are becoming more and more enthusiastic in sports ${ }^{[2]}$. A survey of Chinese urban and rural residents 'participation in physical training in 2007 shows that the proportion of Chinese's participation in physical exercise is on the rise $^{[3]}$. In 2016, the CPC Central Committee and the State Council issued the "Healthy China 2030" plan outline, and elaborated the Chinese health index. The plan outline points out that by 2030, the basic literacy level of the residents will be raised to $30 \%$, that the number of people who participate regularly in physical exercise will soar from 360 million of 2014 to 530 million, that the $3^{\text {rd }}$ public sports facilities network will be basically completed in county rural areas, and that per capita stadium area will be not less than 2.3 square meters. Meanwhile, 15-minute fitness circle coverage will be achieved in the urban communities ${ }^{[4]}$.

In order to improve people's physical quality, the government departments vigorously develop public sports venues, but the sports facilities are relatively simple. For the open-air stadium rest facilities, humanized design is more vacant. It is necessary to take the human nature as one of the design principles of sitting-type furniture that is the necessary and frequently-used facility for the stadium.

\section{Status Quo of Research on the Facilities of Sports Stadiums}

Public rest facilities are the most common and basic furniture. Seats are indispensable public facilities for people, and are the main components of outdoor public rest facilities ${ }^{[5]}$. The design of the rest facilities is an important factor in encouraging people to use the outdoor environment ${ }^{[6]}$. Therefore, people's attention to the design of rest facilities has been gradually improved in recent years. However, there is still problem of imperfect functioning facilities and rather unitary function 
accompanied with the lack of scientific design. And there is problem of humanistic care existed in the design and environmental space needs.

Stadium as an integral part of public space, people are focused on the scale of sports venues and sports facilities, while neglecting the rest facilities which play an important role in the sports stadiums. Most stadiums take account of cost and environmental conditions. Some stadiums are not set up rest facilities. Some other stadiums, the rest facilities are mainly built with stone stools or cement. In those more formal sports venues, there is a special audience chair, but it can only meet the basic "sit" function, let alone that the materials and functions are way too simplex.

\section{Analysis of the Influence of the Rest Facilities on the Users in the Stadium}

Rest facilities are for people to serve, and therefore first to consider is the type of person to be targeted. In consideration of different environments, different users have different use demands. In different environmental conditions, the use of rest facilities will have different effects on the psychological and physiological aspects of the users.

\subsection{Physiological Effects of Rest Facilities on the Users of the Stadium}

Stadium as the recreational fitness place, people's activity mainly divides into the movement, watching competitions, etc. The design of the rest facilities in the stadium is closely related to human physiology and psychology, including the body, five senses and other factors. Physiological aspects include the usage behavior, convenience, labor-saving, and so on. Psychological aspects include color temperature material on the psychological impact of people, psychological satisfaction of convenient use, and so on. Most of the seats are able to rely on rest. At this stage, the material is of great comfort, but of high thermal conductivity. High temperature weather as in the summer, shading and other factors need to be considered to allow people to maintain physiological comfort in the use of rest facilities ${ }^{[7]}$.

\subsection{Psychological Effects of Rest Facilities on the Users of the Stadium}

People's feelings about the environment can not be logically reasoned only by intuition, or can only respond to space by personality and psychological needs. According to the study of human behavior psychology, people have the habit of enjoying the scenery[8]. Therefore, when people choose seats in stadiums, people will select chairs where they can observe the surrounding very well, and the use frequency of those chairs is relatively high. The placing distance of rest facilities will have a certain impact on people's sense of domain, security and interaction among people.

\section{Humanized Design for the Rest Facilities of Outdoor Gymnasium}

\subsection{Humanized Design Principle}

"Designed for human rather than product" was the basic principle of industrial design at the beginning of this century. "Human-oriented" is a rational concept conforming to the requirements of the times, and a guiding ideology for the development of product design in the right direction[9]. Therefore, the "people-oriented" thinking should be closely focused to carry out the design of rest facilities. The category of facility users mainly includes: athletes, spectators and staff. For the athletes, it is to achieve the highest efficiency in the short period of rest to restore physical strength and to adjust the mentality. It is also to achieve, in the next game, more stable play; For the audience, it is to provide a comfortable environment to watch the game in a comfortable state during the whole game; For staff, it is to maintain a high standard of working efficiency and under various conditions, to avoid the impact of mistakes in the work. At the same time, the selection of materials and other issues should also be taken into account for the outdoor stadiums and gymnasiums. The design is mainly embodied in its functionality, safety and environmental protection, etc. 


\subsubsection{Functionality, Convenience and Comfort}

First to meet for rest facilities is their use functions. The use functions are mainly divided into three levels: the basic use function; the extension of subtle functions, including built-in functions and external functions; amenity design based on Ergonomics[10]. In terms of functional design, designers should take comfort and convenience as the basic point and meet other functions while satisfying the basic use function. Standing in users' shoes, designers should also take as the design point of a comfortable environment in open air for the users to relax muscle, to ease fatigue, to store items and to cope with emergencies. As a result, more efficient functions appear. The moment adapting to the development process of humanized design, the rest facilities' practicability and use value are promoted.

\subsubsection{Modeling Conciseness, Structural Security}

Sports venue rest facilities belong to the mass production of practical products. so endeavor should be done to maintain the shape of the smooth line and of concision, but also to meet the modern people' pursuit of simplicity. The addition of many functions on the original foundation exert some influence to the internal structure and appearance of the rest facilities, so the connection between the components should be fully considered in the design and a stable structure should be formed. While fulfilling the functions, the design should also ensure the facilities' safety and convenience, ensure people's safety, and enable people to grasp various structural transformation ways easily and quickly.

\subsubsection{Low-carbon Materials, Eco-environmental Greenness}

Under the guidance of "people-oriented" concept, material is a vital aspect in humanized design. The texture and properties of the material not only affect the appearance of the furniture, but also have different influences on the physical and mechanical characteristics of the furniture. When selecting materials, designers should take full account of its impact on the environment. To improve ecological capacity, Low-carbon materials are the first to think about. Due to the open-air environment, weather conditions should be taken into consideration. And materials with good tolerance and easy-cleaning feature should be selected. Besides, provided that users often sweat when they enjoy games, permeability and heat dissipation are also indispensable factors in choosing materials. The aims are: to enhance people's psychological and physical enjoyment in the short-term use of rest facilities; to attract more people to use; to improve the practical values of the facilities; to promote environmental awareness while arousing people's desire for sports recreation.

\subsection{Demonstration of Humanized Design}

\subsubsection{Referee Chair}

Generally, the referee chair on the court is simple-structured and single-functioned. In the open-air environment, the summer heat impact on the referees' physiology and psychology causes certain influence, while the glare of the sun light to a certain extent affects the referees' judgment of the game process. In the summer, the referee chair, because of prolonged exposure to the sun, will give people a scorching touch, while in the winter, it's cold feeling. At the same time, usually there is no auxiliary device in the referee's chair. The referees sit on the middle line and may make a miscarriage of justice because of the surrounding environment. 

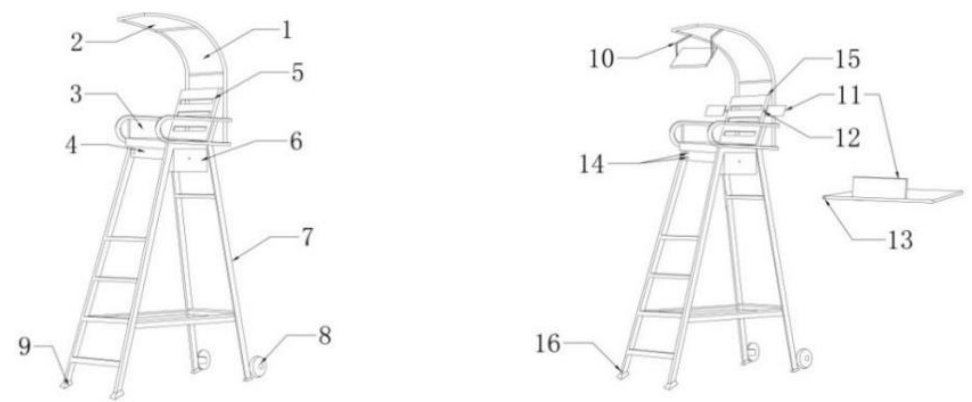

Fig. 1 Referee Chair

1.sunshade shed 2. sunshade board 3. work panel 4.electric storage device and refrigeration heating device 5 . chair back 6 . storage box under the seat

7. chair leg 8.wheel 9. anti-slide chair leg 10. adjustable connection structure

11. mirror 12. connection hinge 13 . work panel with handrail rotating shaft

14. switch 15 . water injection port 16 . water outlet

In view of the above problems, the referee chair is designed for the open-air stadium. The chair, the sitting face, the armrest and the back of the chair are all hollow structures, with pipes inside which can be injected by water injection. Secondly, the bench is provided with an electric storage device and a refrigeration heating device, which can be used to adjust the temperature of the water in the pipe, avoiding the searing touch of the seat in the hot summer and making the seat warm in the cold winter. The power of the refrigeration heating device is supplied by solar energy collected from the solar panels on the surface of the sunshade. The top of the chair is shaded with sunshade shed and board which are used to shield the eyes from direct sun. In the back side of the chair, there are small holes which can spray water mist, reduce the temperature around the body, and alleviate the summer heat. The back side of the chair is also provided with a mirror that can be put up with the back of the chair. The use of the mirror can adjust angle by rotating shaft and reflect to the mirror on the work panel. According to the principle of reflection, the mirror increases the referee's perspective and reduces the false positives or false negatives. To avoid the accident on the court, a locker is attached to the floor to store some first aid supplies.

The design, from a human point of view, not only solves the outdoor environment for the referees' physical and psychological discomfort, but fully utilizes the open-air environment, solar energy and low carbon ring. Under the premise of not affecting the normal use of referee chair, the use of reflection principle increases the referees' perspective, so that the referees, in comfortable conditions, can be more concentrated on law enforcement competition, thus improving the accuracy of work.

\subsubsection{Lounge Chair for Athletes}

In the course of the game, the short rest time is very important for the athletes to not only adjust the mentality as far as possible, but also restore the strength coping with the next game. But the common rest chair, only can meet the basic function of sitting, ignoring the athletes' own needs as for durability. 

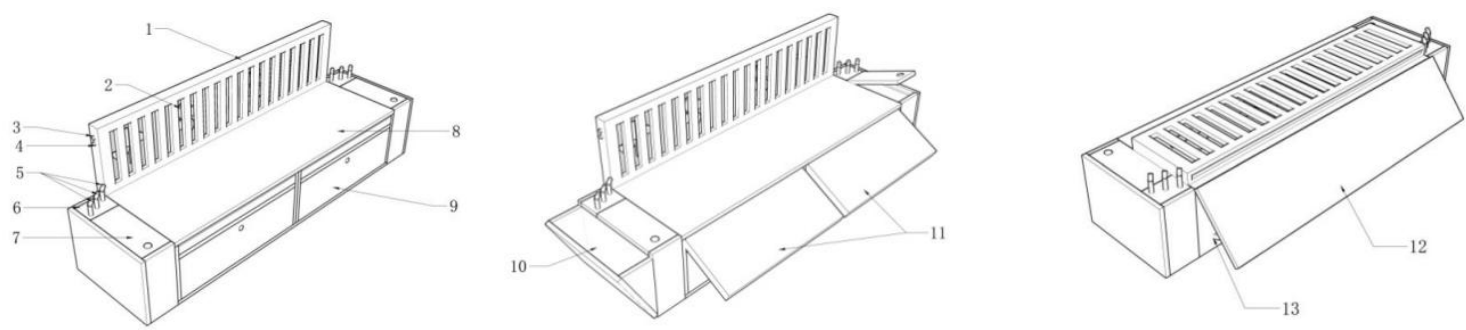

Fig. 2 Lounge Chair for Athletes

1.Chair back 2. Fan 3. Fan switch 4. Massage board switch

5. Spray nozzle 6. Storage tank 7. Mini freezer 8. Seat interface 9. Locker

10. Storage basket 11.Massage board 12. Solar panel 13. Support plate

Considering the physical and psychological conditions of athletes and combining them with the rest chair, the writer designs lounge chair for athletes. On the basis of the seat, the heat dissipation parts, massage relaxation parts and storage parts are added to the chair, and three new functions are gathered into one. But due to their modularization, they are properly incorporated into the seat interior space. The design helps the athlete to not only dissipate heat quickly and calm the mood, but also relax the tense muscles during the break. Athletes in the exercise process will sweat a lot, so they need to replenish water. Therefore, staff need to put it into a small freezer in advance. It is convenient for athletes to take, while reducing the workload of staff.

\subsubsection{Audience Chair}

Nowadays with the gradually improved life quality, watching on-site games has grown popularity in people. The audience, as an important part in the process, has also been of great significance for the scene atmosphere and impact on the game players. While people advocating humanization, more attention should be still focused on the stadium audience chair. In this public occasion, Unremitting efforts should be done to settle the cleaning problem and to think about people's feeling. Only in this way can the audience enjoy the game in the most comfortable environment. So the audience chair is designed.
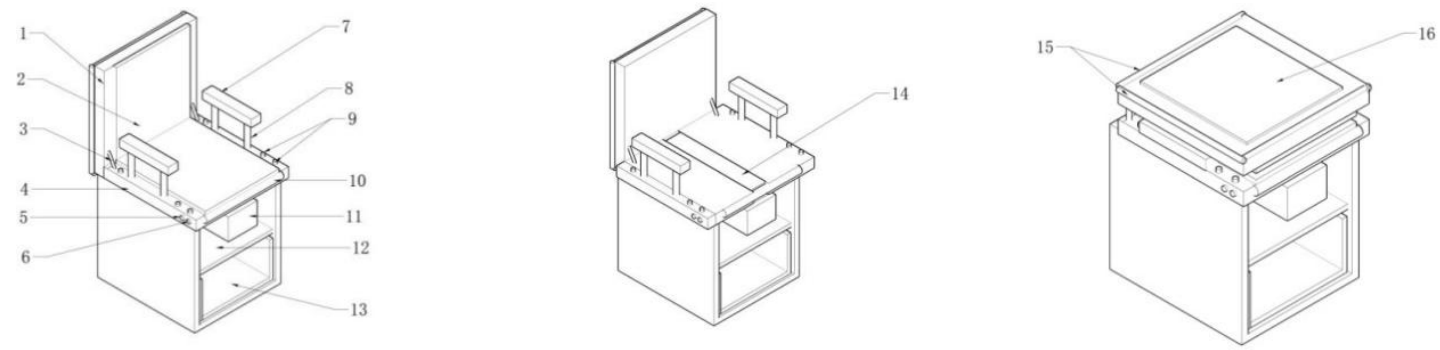

Fig. 3 Audience chair

1. chair back 2. soft package 3. connecting parts 4. seat interface

5. refrigeration device switch 6. spray switch 7. Handrail 8.contraction support lever

9. nozzle 10. Shaft 11.refrigeration device and accumulators 12. storage box

13. garbage chute 14 . inline board 15 . covering cloth 16 . solar panel

The design is divided into the daily use of summer and winter. In hot summer, equipped with refrigeration and spray devices, the audience chair can ease the heat of the audience, so that the audience have a happy mood to watch the game. In cold winter, covering the soft package on the surface avoids the icy touch. Due to the limited space, there is problem of goods placing and littering. The design makes full use of the lower space of the seat. Down the chair there is a storage box and a garbage box. The design solves the problem of the goods placing, and facilitates the cleaners to clean the rubbish. Because of the large size of the site, not all users' needs can be taken care of. Insufficient 
space phenomenon appears if the larger audience sit in the chairs. The design makes full use of space between adjacent seats, where seats can be opened from the middle stretch embedded board, widening seat interface area to increase user comfort. Finally, the design adopts folding mode. When not in use, it can cover itself and avoid sun and rain, so as to prolong the service life.

\section{Conclusions}

The main point of the design of the open-air gymnasium rest facilities is to take full account of environmental factors. According to the different roles of users, different requirements, which combine function, security and environmental protection, make the facilities more targeted, enhance the added value, and provide comfortable experience for users in the process of use.

In the tide of national fitness, the rest facilities of sports venues play a very important role. It not only is the seats, but also shoulder the responsibility to attract more people to join the movement. Humanized design can improve the enjoyment of people in the process, thus stimulating people's desire to exercise, and improving the game's seating rate as well. Therefore, it is necessary to increase the attention to the facilities of stadiums and to integrate humanization into the design.

\section{Acknowledgments}

The project was supported by Key Laboratory of Wood Industry and Furniture Engineering of Sichuan Provincial Colleges and Universities, Sichuan Provincial Philosophy Social Sciences Key Research Base of Modern Design and Culture Research Center (Grant No. MD15E017).

\section{References}

[1] Jianlong Wang, Study on the influence of physical exercise on the mental health of citizens, Hubei Sports Science and Technology, 2015, 6, pp. 519-522.

[2] Meng Li, A study on the Trend of Sports Populace, Modern Sports Science and Technology, 2016, 14, pp. 94-96.

[3] China Mass Sports Research Group, Investigation and Research on the Present Situation of Mass Sports in China , Beijing Sports University Press, 2005, 4, pp. 214-228.

[4] State Council Health Reform Leadership Group, 'Health China 2030’ Plan Outline, The National Symposium on Chinese Medicine for the Treatment of Disease-preserving and Rehabilitation and the 2016 Annual Session of the Committee, 2016.

[5] Yunfeng Ling, Study on Public Seating Facilities, $\mathrm{Xi}$ ' an University of Architecture and technology(2007)

[6] Dongxia Su, Xia Ren, Outdoor Seating Facilities Design Research Planner, 2001, 1, pp. 87-90.

[7] Xiuting Zhang, User-Friendly Design of Urban Public Facilitie, Qingdao Technology University (2012)

[8] Jing Wang, Full Analysis and Extension of Humanized Furniture Design, Hefei University of Technology(2006)

[9] Shajun Lu, Research on Humanized Design of Outdoor Furniture in City, Jiangnan University(2004)

[10] Shengzu An, Xiaoyan Zhang, Zhijun Liu, Humanized Design of Furniture, Furniture and Interior Decoration, 2005, 6, pp. 50-51. 